\title{
NaviAirway: a Bronchiole-sensitive Deep Learning-based Airway Segmentation Pipeline for Planning of Navigation Bronchoscopy
}

This paper was downloaded from TechRxiv (https://www.techrxiv.org).

\section{LICENSE}

CC BY 4.0

SUBMISSION DATE / POSTED DATE

24-02-2022 / 03-03-2022

\section{CITATION}

Wang, Andong; Tam, Terrence Chi Chun; Poon, Ho Ming; Yu, Kun-Chang; Lee, Wei-Ning (2022): NaviAirway: a Bronchiole-sensitive Deep Learning-based Airway Segmentation Pipeline for Planning of Navigation Bronchoscopy. TechRxiv. Preprint. https://doi.org/10.36227/techrxiv.19228296.v1

$\mathrm{DOI}$ 


\title{
NaviAirway: a Bronchiole-sensitive Deep Learning-based Airway Segmentation Pipeline for Planning of Navigation Bronchoscopy
}

\author{
Andong Wang, Terence Chi Chun Tam, Ho Ming Poon, Kun-Chang Yu, and Wei-Ning Lee
}

\begin{abstract}
Navigation bronchoscopy is a minimally invasive procedure in which doctors pass a bronchoscope into a subject's airways to sample the target pulmonary lesion. A three-dimensional (3D) airway roadmap reconstructed from Computer Tomography (CT) scans is a prerequisite for this procedure, especially when the target is distally located. Therefore, an accurate and efficient airway segmentation algorithm is essential to reduce bronchoscopists' burden of pre-procedural airway identification as well as patients' discomfort during the prolonged procedure. However, airway segmentation remains a challenging task because of the intrinsic complex tree-like structure, imbalanced sizes of airway branches, potential domain shifts of CT scans, and few available labeled images. To address these problems, we present a deep learning-based pipeline, denoted as NaviAirway, which finds finer bronchioles through four major novel components - feature extractor modules in model architecture design, a bronchiolesensitive loss function, a human-vision-inspired iterative training strategy, and a semi-supervised learning framework to utilize unlabeled CT images. Experimental results showed that NaviAirway outperformed existing methods, particularly in identification of higher generation bronchioles and robustness to new CT scans. On average, NaviAirway takes five minutes to segment the CT scans of one patient on a GPU-embedded computer. Moreover, we propose two new metrics to complement conventional ones for a more comprehensive and fairer evaluation of deep learningbased airway segmentation approaches. The code is publicly available on https://github.com/AntonotnaWang/ NaviAirway.
\end{abstract}

Index Terms-Airway segmentation, Computed Tomography $(\mathrm{CT})$, semi-supervised learning, deep learning training strategy

This project was in part supported by COVID-19 Action Seed Funding of Faculty of Engineering, The University of Hong Kong. Wei-Ning Lee is the corresponding author.

Andong Wang is with the Department of Electrical and Electronic Engineering, The University of Hong Kong, Hong Kong, China (email:wangad@connect.hku.hk).

Terence Chi Chun Tam is with Respiratory Division, Department of Medicine, The University of Hong Kong, Hong Kong, China, and also with Queen Mary Hospital, Hong Kong, China (email:tcctam@netvigator.com).

Ho Ming Poon is with the Department of Electrical and Electronic Engineering, The University of Hong Kong, Hong Kong, China (email:hmpoon6@connect.hku.hk).

Kun-Chang Yu is with Broncus Medical, Inc., San Jose, CA, 95134 USA (e-mail:jyu@broncus.com).

Wei-Ning Lee is with the Department of Electrical and Electronic Engineering, The University of Hong Kong, Hong Kong, China, and also with the Biomedical Engineering Programme, The University of Hong Kong, Hong Kong, China (e-mail:wnlee@eee.hku.hk).

\section{INTRODUCTION}
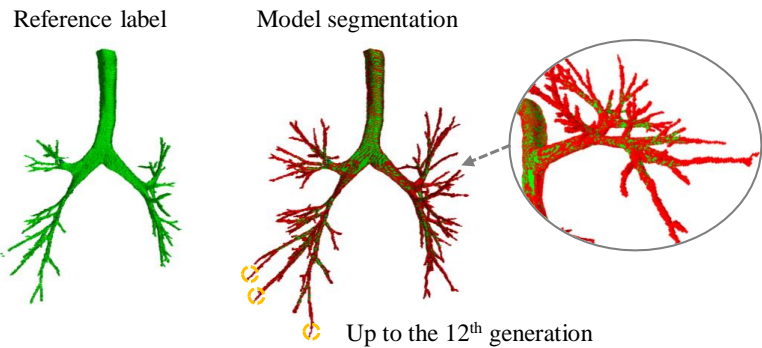

Green: overlapped volume

Red: extra airway voxels found by the model

Fig. 1. NaviAirway finds more finer bronchioles than the reference label.

O OMPUTED Tomography (CT) is a predominant medical imaging modality for the assessment of lung diseases, such as lung cancer and chronic obstructive pulmonary disease (COPD). If a nodule is observed in the CT images, lung biopsy can be further performed to determine whether the nodule is benign or not. Among available lung biopsy procedures, navigation bronchoscopy (NB) is the safest and superior for accessing peripheral pulmonary lesions [1]. For better procedural efficiency and patient care, NB requires a pre-planned 3D airway roadmap segmented and reconstructed from CT images. Then, NB guides doctors to navigate the bronchoscope down into the bronchioles for target nodule sampling according to the roadmap [2]-[4]. Accurate and efficient airway segmentation shall thus greatly reduce bronchoscopists' burden of pre-procedural airway identification as well as patients' discomfort during the procedure.

However, accurate and automated airway segmentation from CT images remains to be challenging primarily because of 1) morphological complexity of airways, 2) imbalanced sizes between airways and background as well as between lowand high-generations airways in $\mathrm{CT}$ images, 3) the potential domain shift of CT images across different machines and different patients, and 4) few labeled images available and a large number of unlabeled images.

1) The first challenge comes from the most prominent characteristic of airways - the complex tree structure, which begins from the trachea and ends at the alveoli. The trachea beginning at the larynx is denoted as Generation 0 , while the divided left and right main-stem bronchi as Generation 

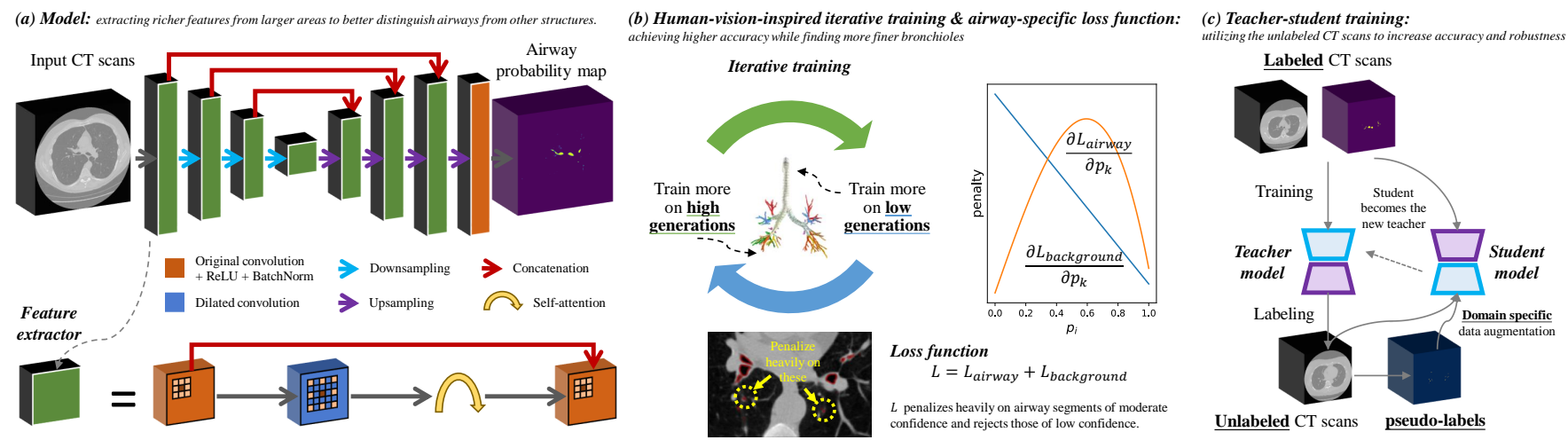

Fig. 2. The pipeline of NaviAirway. (a) Model architecture. (b) Proposed iterative training strategy and loss functions. Graphs on the right show the intuition of the proposed loss function: penalizing heavily on airway segments of not high confidence and ignoring those of too low confidence. (c) The structure of our semi-supervised learning approach named teacher-student training.

1. After that, the airways become finer until the 23rd generation-alveolar sacs [5]. In our paper, low generation stands for large airways closer to the trachea, while high generation refers to fine bronchioles closer to the alveolar sacs. 2) The second challenge comes from the fact that the airways only take up a small fraction in CT images. It is difficult even for human experts to exclude all background information and target the airways accurately. Moreover, unlike humans, who can recognize airway branches as geometric shapes, existing computer algorithms can only identify them pixel-wise and thus often overlook fine bronchioles of few-pixel thickness. 3) The third challenge concerns algorithm robustness and usability when dealing with new image datasets. It is possible that an airway segmentation algorithm only learns special airway features, instead of the general ones, from the given CT scans. When tested with new CT scans, the performance of the algorithm may drop drastically, thus losing generality. 4) The fourth challenge is a frequent problem in medical image segmentation. It is impractical to create a large labeled medical image dataset due to the high labeling cost. Employing unlabeled images will be clinically significant but is lacking.

Over the years, many airway segmentation methods have been developed. There are two main categories - traditional methods which rely on manually selected features [6]-[19] and deep learning-based methods which combine Convolutional Neural Networks (CNNs) with traditional methods or focus on new model architecture design [20]-[36]. However, most methods were designed to deal with the first (i.e., complex tree structure of airways) and the second challenge (i.e., imbalanced airway-background size). Yet, the third and the fourth challenges concerning data distributions have not been well addressed. The two latter challenges may not be an issue for traditional approaches as they rely more on manually defined airway features. However, they are crucial for deep learningbased methods as deep learning automatically extracts target features from the data. Specifically, deep learning method development involves three components: model, training, and data. The model design was the main focus in most earlier studies, whereas alternative training strategies have not been fully explored. Furthermore, previous methods overlooked the large amount of unlabeled but informative data, from which the model could learn much richer features of airways to greatly improve the segmentation accuracy and model robustness.

To address the gap and tackle the challenges, we present a novel method, coined as NaviAirway. We design a new feature extractor module, an airway-specific loss function, and a human-vision-inspired training strategy to tackle the first two challenges-morphological complexity and imbalanced sizes. For the third and the fourth challenges, which concern data, we propose and incorporate a semi-supervised learning approach to increase the model accuracy and model robustness.

The backbone model of NaviAirway is based on a 3D UNet [20] and equipped with a new lightweight feature extractor module that combines dilated convolution and self-attention mechanism. It can extract richer features from larger areas to segment airways. During model training, the proposed loss function pushes the model to recognize more bronchioles of higher generations. At the same time, the proposed humanvision-inspired iterative training strategy guides the model to specifically extract features of both low and high generation airways and preserve those learned features. To use unlabeled CT images, we develop a semi-supervised learning approach based on teacher-student training [37] which learns the intrinsic distribution of chest CT images to achieve a higher segmentation accuracy than the supervised learning approach. During model testing, unconnected shapes are discarded from the airway tree at the model output. To evaluate model performance, we first test NaviAirway on two public datasets and compare its performance with recent state-of-theart methods. The comparison results show that our method is more accurate and detects longer airway trees than existing methods. Moreover, the computational cost of NaviAirway is deemed acceptable for navigation bronchoscopy as our method on average takes five minutes to process the CT scans of one patient. We subsequently test NaviAirway on a private dataset, demonstrating its robustness to a previously unexposed dataset.

In addition to the method itself, evaluation metrics also play a vital role in the development process. Many studies followed the metrics defined in the EXACT'09 Challenge [8]. However, the metrics in [8] are essentially evaluation standards for traditional methods and may not be suitable for deep learning-based methods. It is because, in the airway 
segmentation task, the airway labels are not $100 \%$ correct and may miss some or even a substantial number of bronchioles. In particular, we only have "reference" labels, instead of "ground truth" labels, which can only be obtained when the actual bronchoscopy procedure is eventually performed. Hence, in cases of airway segmentation, the goal of a deep learning model is not to provide airway segments that perfectly match the reference; instead, the deep learning model is to learn from the reference and apply the knowledge of airway features back to the raw CT scans to find all recognizable bronchioles. It is expected that the deep learning model can do even better than the reference. Therefore, we additionally standardize existing metrics and propose new metrics to have a fairer and more comprehensive evaluation of deep learning-based airway segmentation methods.

Our contributions are summarized as follows:

- A more accurate and more robust airway segmentation pipeline that is optimized in a more comprehensive manner, including the model, the loss function, the training strategy, and the utilization of unlabeled data.

- A simple but effective feature extractor module that extracts morphological features in a larger area and can better distinguish airways from other structures.

- A new loss function which drives the model to recognize finer bronchiole or more generally fine and long tubular shapes in other scenarios.

- A new human-vision-inspired iterative training strategy that guides the model to learn both the features of fine and coarse airways, while preventing knowledge loss of airway features.

- The first attempt to propose semi-supervised learning - teacher-student training which incorporates domain knowledge of airways in pseudo annotation generationfor improved accuracy and robustness of airway segmentation with unlabeled chest CT images.

- A new framework of metrics for a fairer and more complete evaluation of deep learning-based airway segmentation methods.

\section{Related Works}

\section{A. Traditional Methods}

Traditional methods mainly include 1) region growing and thresholding, 2) morphologic and geometric model-based methods, and 3) hybrid approaches combining the above two methods [6], [7]. For example, EXACT'09 Challenge [8] presented 15 airway tree extraction algorithms submitted to the competition. Ten out of the 15 methods used region growing and thresholding techniques which utilize brightness of different tissues. Similar techniques were also developed, including pixel value filtering and thresholding [9], thresholding and rectangular region mask [10], GVF snakes [11], fuzzy connectivity [12], [13], and two-pass region growing [14]. Alternatively, airways were mathematically defined according to their morphologic and geometric features of airway for extraction [15], [16]. Hybrid methods combined the strengths of the two to provide better segmentation [17]-[19].

\section{B. Deep Learning-based Methods}

Compared with traditional methods, deep learning-based models, on average, detect twice longer airways [21], [22], [25]-[27], [30], [33], [38]-[40]. Abundant studies combined Convolutional Neural Networks (CNNs) and traditional methods based on the idea that CNN provided preliminary results, and the traditional method was responsible for refinement. One mainstream of studies used 3D U-Net [20] as the backbone model and built different post-processing approaches, which included fuzzy connectedness region growing and skeletonization guided leakage removal [21], image boundary postprocessing to minimize the boundary effect in airway reconstruction [22], and freeze-and-grow propagation [23]. Besides, other works focused on designing the backbone network. A simple and low-memory 3D U-Net was proposed in [24]. Graph Neural Networks (GNNs) were adopted to segment airways [25], [26]. In [27], a GNN module was incorporated into a 3D U-Net, while [28] developed a graph refinementbased airway extraction method by combining GNN and mean-field networks. Beyond utilizing existing deep learning models which were built for general tasks, in more recent studies, new network architectures (usually based on 3D UNet) considering special features of airways were designed to achieve a higher segmentation accuracy. They included patch classification by $2.5 \mathrm{CNN}$ [29], AirwayNet [30], Airway-SE [31], 2D plus 3D CNN [32], attention distillation modules plus feature recalibration modules [33], [34], group supervision plus union loss function [35], and attention on weak feature regions [36].

\section{Method}

Consider a labeled set $\mathcal{D}:\left\{\left(\boldsymbol{x}_{i}, \boldsymbol{a}_{i}\right)\right\}_{i=1}^{N}$, where $\boldsymbol{x}_{i}$ is a $3 \mathrm{D}$ CT image, $\boldsymbol{a}_{i}$ is the corresponding airway annotation map (which denotes each voxel on $\boldsymbol{x}_{i}$ as either background or airway), and $N$ is the number of labeled images. Also, we have an unlabeled set $\mathcal{U}:\left\{\boldsymbol{x}_{i}\right\}_{i=1}^{M}$, where $M$ is the number of unlabeled images and $M \gg N$.

Our goal is to build a bronchiole-sensitive segmentation pipeline trained on $\mathcal{D}$ and $\mathcal{U}$. There are five main components in the pipeline (Figure 2), which are a backbone model $\Phi_{\theta}$ with proposed feature extraction module (Section III-A), a new bronchiole-sensitive loss function (Section III-B), a human-vision-inspired iterative training strategy (Section IIIC), teacher-student training using unlabeled images to increase model robustness (Section III-E), and simple post-processing (Section III-E).

\section{A. Model: Feature Extraction in A Large Area}

We built our model based on the structure of 3D U-Net. To avoid the interference from other tubular shapes, such as the esophagus and compared with the conventional convolution kernel, our model used features from a larger surrounding area to decide whether the pore-like structure was part of the airway. Hence, we introduced a feature extractor module which consisted of one dilated convolution, one self-attention block, and two typical convolutional kernels (Figure 2(a)). Several feature extractors (the green rectangles in Figure 2(a)) then 
fused together with down-sampling (pooling) and up-sampling (deconvolution) steps to constitute the model.

The reason we adopted dilated convolutions was that they enlarged the feature extraction area without increasing the size of the model. If all the convolutional kernels had been typical ones, the receptive field size $r_{0}$ of the feature map $\Phi_{\theta}^{l}\left(\boldsymbol{x}_{i}^{j}\right)$, an intermediate result after the $l$-th convolutional layer, would have been [41]

$$
r_{0}=\sum_{p=1}^{l}\left(\left(k_{p}-1\right) \prod_{q=1}^{p-1} s_{q}\right)+1
$$

where $k_{p}$ is the size of the $p$-th kernel and $s_{q}$ is the stride of the $q$-th kernel. Note that the first convolutional kernel means the kernel processing the input image. Therefore, the $l$-th convolutional kernel processes the result given by the $(l-1)$ th kernel.

In our study, by introducing dilated convolutions, the size of the receptive field became

$$
r_{0}^{\prime}=\sum_{p=1}^{l}\left(k_{p}^{\prime} \prod_{q=1}^{p-1} s_{q}\right)+1,
$$

where $k_{p}^{\prime}=d_{p}\left(k_{p}-1\right)$ was for dilated convolutions with $d_{p}$ being the dilation spacing between kernel elements, and $k_{p}^{\prime}=k_{p}-1$ for other typical convolutions. As shown in Figure 2 (a), the dilation spacing was 1 .

We can see that the new receptive field size $r_{0}^{\prime}$ was approximately $\prod_{p \in D} d_{p}$ times larger than the original $r_{0}$, while the model size remained the same. In other words, the area for feature extraction in our proposed scheme was $\prod_{p \in D} d_{p}$ times larger than the original one. Note that D stands for the positions of dilated convolutions before the $l$-th kernel. For instance, when $d_{p}=4$, for the 2 nd dilated convolution, its receptive field size is 8 times larger than the scenario where only conventional convolutions are used. Therefore, our model learned the CT image features from an eight times larger surrounding area.

The self-attention we incorporated into the model was inspired by Squeeze-and-Excitation Network [42]. It could further improve the model performance by emphasizing the areas where the model should focus on while deemphasizing the minor regions.

\section{B. Loss Function: A Search for More Bronchioles of Higher Generations}

Based on dice loss, a new loss function $\mathrm{L}$ has been formulated to let the model recognize more bronchioles of higher generations, which are of small diameters but essential for navigational bronchoscopy. The proposed model outputs two prediction maps of airway and background, respectively. Therefore, L consists of two components (Equation (3)) to deal with airway and background voxels differently (Equation (4) and (5)):

$$
L=L_{a w}+L_{b g}
$$

$$
\begin{aligned}
& L_{a w}=1-\frac{2 \sum_{k=1}^{K} p_{k}^{2} a_{k} w_{k}}{\sum_{k=1}^{K} p_{k}^{4}+\sum_{k=1}^{K} a_{k}^{2}}, \\
& L_{b g}=1-\frac{2 \sum_{k=1}^{K} p_{k} a_{k} w_{k}}{\sum_{k=1}^{K} p_{k}^{2}+\sum_{k=1}^{K} a_{k}^{2}},
\end{aligned}
$$

where subscripts $a w$ and $b g$ denote airway and background, respectively; $k$ denotes voxel position; $K$ is the total number of voxels; $p_{k} \in[0,1]$ is the model confidence of a voxel being background or airway, while $a_{k} \in 0,1$ is the computer-assisted manual annotation of the $k$-th voxel, where the voxel being background is assigned as 0 and the voxel being the airway is given a $1 . w_{k}$ is a weight value proportional to the ratio of the number of annotated airway voxels to the number of annotated background voxels.

Different from conventionally used dice loss function, $L_{a w}$ replaces model prediction confidence $p_{k}$ with pseudoconfidence $p_{k}{ }^{2}$. The reason of using pseudo-confidence is analogous to the scheme where athletes bear extra weights during training and release those weights in competitions. When $p_{k}$ is 0.5 , the pseudo-confidence is only 0.25 . Hence, the model must learn harder to push $p_{k}$ to be larger than 0.7 , which means the pseudo-confidence of being airway can be larger than 0.5. However, during model inference, we only used $p_{k}$ to decide airway segments. With the above training approach, our model could recognize more fine bronchioles than the one with dice loss function solely.

The derivative of $\mathrm{L}$ helps elaborate the mechanism of the proposed loss function. For $p_{k}$ on the airway prediction map, $\frac{\partial L}{\partial p_{k}}=\frac{\partial L_{a w}}{\partial p_{k}}$, while on the background prediction map, $\frac{\partial L}{\partial p_{k}}=\frac{\partial L_{b g}}{\partial p_{k}} . \partial p_{k}$ follows a linear pattern, whereas $\frac{\partial L_{a w}}{\partial p_{k}}$ penalizes heavily on airway segments of moderate confidence and rejects those of too low confidence.

$$
\begin{gathered}
\frac{\partial L_{a w}}{\partial p_{k}}=\frac{-4 p_{k} a_{k}\left(\sum_{l=1}^{K} p_{l}^{4}+\sum_{l=1}^{K} a_{l}^{2}\right)+8 p_{k}{ }^{3}\left(\sum_{l=1}^{K} p_{l}{ }^{2} a_{l}\right)}{\left(\sum_{l=1}^{K} p_{l}{ }^{4}+\sum_{l=1}^{K} a_{l}^{2}\right)^{2}}, \\
\frac{\partial L_{b g}}{\partial p_{k}}=\frac{-2 a_{k}\left(\sum_{l=1}^{K} p_{l}^{2}+\sum_{l=1}^{K} a_{l}^{2}\right)+4 p_{k}\left(\sum_{l=1}^{K} p_{l} a_{l}\right)}{\left(\sum_{l=1}^{K} p_{l}^{2}+\sum_{l=1}^{K} a_{l}^{2}\right)^{2}}
\end{gathered}
$$

\section{Training Strategy: Solution to Two Imbalanced Distributions in Annotation}

There are two imbalanced distributions in airway annotation. One is that airway segments only take a small fraction of space compared with the background. The other is that airway segments of high generations have a larger number but take up fewer voxels compared with airways of low generations. To address such issue, we proposed a new training strategy as shown in Figure 2 (b).

Unlike existing methods following a first-low-generationthen-high-generation training scheme, our model is trained on the two kinds of generations iteratively. We first crop the training images to cuboids. For training cuboid pairs $\left(\boldsymbol{x}_{i}^{j}, \boldsymbol{a}_{i}^{j}\right)$ (i.e., the $j$-th cuboid pairs of the $i$-th chest CT image), we define a value $t_{i}^{j}$ (ratio of the number of outermost voxels of the airway segment to the total number of airway voxels) to 
quantify the size of the airway in $\boldsymbol{a}_{i}^{j}$. When training focuses more on high generations, the probability of a $\left(\boldsymbol{x}_{i}^{j}, \boldsymbol{a}_{i}^{j}\right)$ chosen in a batch is proportional to $t_{i}^{j}$. In contrast, when training focuses more on low generations, the probability value is inversely proportional to $t_{i}^{j}$. When a cuboid $\boldsymbol{x}_{i}^{j}$ contains no airway voxel, its probability is assigned a constant $\delta$ (In our experiments, $\delta$ is small because the model should focus on areas containing airways). The conditions for probability values are listed in Equation (8):

$$
p\left[\left(\boldsymbol{x}_{i}^{j}, \boldsymbol{a}_{i}^{j}\right)\right]=\left\{\begin{array}{rc}
\beta t_{i}^{j} & \left(t_{i}^{j} \neq 0, \text { more on high generations }\right) \\
\frac{1}{\beta t_{i}^{j}} & \left(t_{i}^{j} \neq 0, \text { more on low generations }\right) \\
\beta & \left(t_{i}^{j}=0\right)
\end{array}\right.
$$

where $\beta$ is a manually selected scalar (by try and error) to control the scale of the probability values.

Why is our proposed iterative training better? It is inspired by how humans observe an image. If a person only looks at the overall information in an image, details may be overlooked. Similarly, training on all cuboids equally will lead to poor segmentation of fine airways. The standard first-low-generationthen-high-generation training strategy make improvements by enabling subsequent detection of details after a gross view. However, a person does not pay attention to details only once. Instead, a person observes in a repetitive zoom-in-andzoom-out manner. Specifically, we examine different areas for details and inspect the same area multiple times. This is the underlying principle of our proposed training strategy for a deep learning model to interpret an image better.

\section{Teacher-student Training: Utilization of Unlabeled Images}

Leveraging an unlabeled dataset $\mathcal{U}$, we adopt semisupervised learning to enhance segmentation performance (see Figure 2(c)). Recall that a larger number of unlabeled images than the labeled ones (i.e., $M \gg N$ in the preliminaries section) provides semi-supervised learning with a more complete picture of the raw data distribution [43]. We hereby proposed a semi-supervised learning approach - teacher-student training-based on Noisy Student [37]. In brief, our method used the model that was trained on labeled dataset as a teacher model $\Phi_{\theta}^{T}$ to generate pseudo labels for unlabeled images, and a student model $\Phi_{\theta}^{S}$ was then trained with both labeled and unlabeled images. We used data augmentation to help the student model $\Phi_{\theta}^{S}$ learn by analogy. General data augmentation approaches for medical images, including random flip and affine, random blur, random motion [44], were applied on chest CT scans, while some airway morphology specific data augmentation approaches were applied on airway annotations. Details of the student-teacher training are provided in Algorithm 1

\section{E. Post Processing}

An optimized model $\Phi_{\theta^{*}}$ is thereafter obtained and used to generate an airway confidence map $\Phi_{\theta^{*}}(\boldsymbol{x})$. By setting a threshold $t$, we obtain an airway mask $\boldsymbol{A}=\Phi_{\theta^{*}}(\boldsymbol{x})>t$.

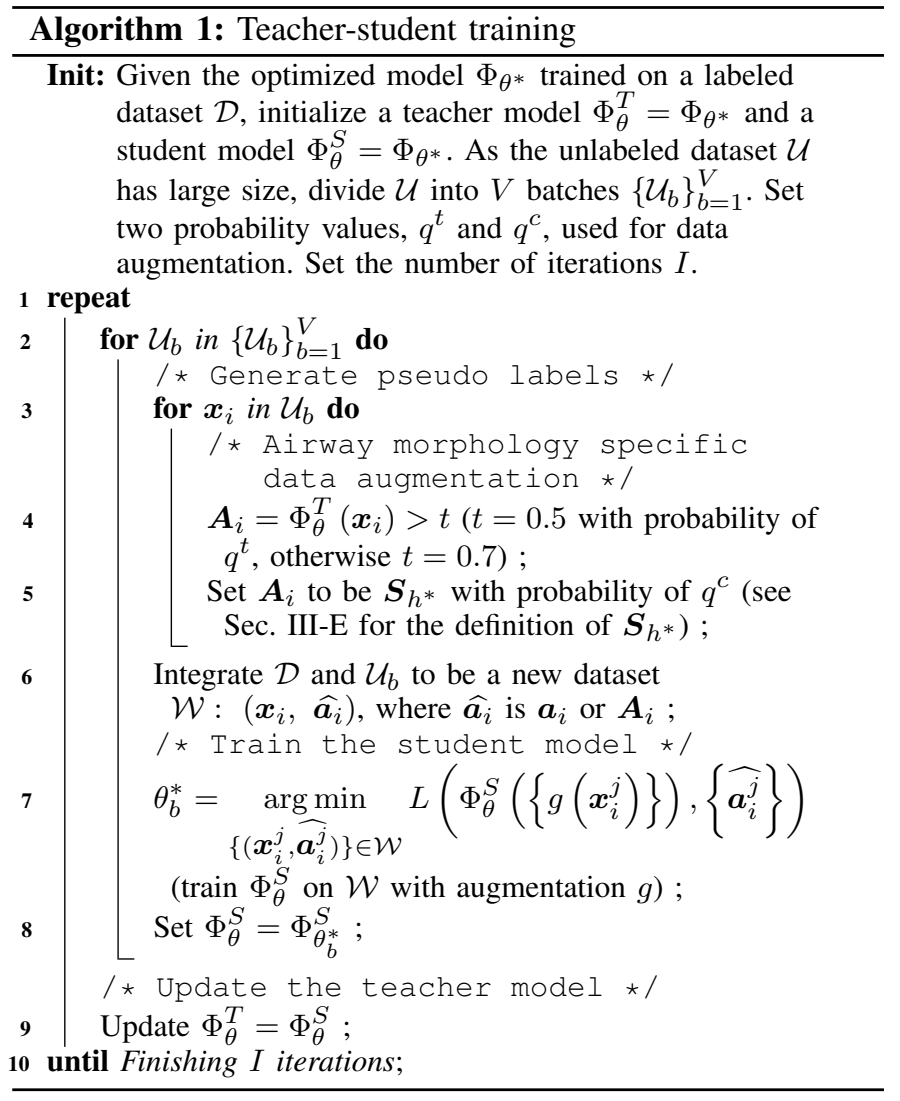

Next, the common sense that airways are connected leads us to find the largest connected shape in the model output. Suppose $\boldsymbol{A}$ consists of several separated shapes $\left\{\boldsymbol{S}_{h}\right\}$, we find $\boldsymbol{S}_{h^{*}}=\max \left|\boldsymbol{S}_{h}\right|$ as the airway segmentation. In addition, there exist some broken airway branches in $\left\{\boldsymbol{S}_{h}\right\} \backslash \boldsymbol{S}_{h^{*}}$. We connect those broken branches to $\boldsymbol{S}_{h^{*}}$ if they are close enough. To connect, we first define a search range $R$. For every end point $e$ of airway branches in $\boldsymbol{S}_{h^{*}}$, if any shape $\boldsymbol{S}_{\hat{h}}$ in $\left\{\boldsymbol{S}_{h}\right\} \backslash \boldsymbol{S}_{h^{*}}$ is within $B_{R}(e)$ (i.e., the neighborhood of $e$ ), update $\boldsymbol{S}_{h^{*}}$ by $\boldsymbol{S}_{h^{*}}:=\boldsymbol{S}_{h^{*}}+\boldsymbol{S}_{\hat{h}}$, and make $\boldsymbol{S}_{h^{*}}$ a connected shape by lowering threshold $t$ within $B_{R}(e)$ to fill the gap. In our experiments, we found that $\boldsymbol{S}_{h^{*}}$ was already good, so connecting broken branches to it was just for refinement, and $R$ was set to be small.

\section{EXPERIMENTS}

\section{A. Datasets}

As shown in Table I two public datasets (EXACT'09 [8] and LIDC-IDRI [45]) and one private dataset (QMH) were used to evaluate NaviAirway. Each case has multiple 2D lung CT images of one patient for 3D reconstruction, and the size of each 2D slice is 512x512 (pixels). Note that both EXACT'09 and LIDC-IDRI did not provide official airway annotations. Twenty cases (CASE1 to CASE20) from EXACT'09 and 40 cases (chosen by slice thickness and pixel spacing) from LIDC-IDRI were annotated using ITK-SNAP and manually corrected by Shanghai Jiao Tong University [34]. After that, the 60 labeled cases (denoted as EXACT'09-TR-LIDC-IDRI) were randomly split into a training set (50 cases) and a testing 
TABLE I

DATASETS USED FOR TRAINING AND EVALUATION.

\begin{tabular}{|c|c|c|c|c|c|c|}
\hline Name of dataset & $\begin{array}{l}\text { Number } \\
\text { of cases }\end{array}$ & $\begin{array}{l}\text { Labeled } \\
\text { cases }\end{array}$ & Labeling method & $\begin{array}{l}\text { Slice thickness } \\
(\mathrm{mm})\end{array}$ & $\begin{array}{l}\text { Pixel spacing } \\
(\mathrm{mm})\end{array}$ & Remark \\
\hline EXACT'09 [8] & 40 & 40 & By ITK-SNAP with experts' manual correction [34] & $0.6-1.25$ & $0.5-0.8$ & Public \\
\hline LIDC-IDRI [45] & 1018 & 40 & By ITK-SNAP with experts' manual correction [34] & $<0.625$ & $0.5-0.7$ & Public \\
\hline QMH & 21 & 9 & By LungPoint with experts' manual correction & $1.0-5.0$ & $0.5-0.9$ & Private \\
\hline
\end{tabular}

TABLE II

THE PROPOSED METRICS FOR MODEL PERFORMANCE EVALUATION.

\begin{tabular}{|c|c|c|c|}
\hline $\begin{array}{l}\text { Metric } \\
\text { category }\end{array}$ & Metric name & Formula & Evaluation aspect \\
\hline \multirow{3}{*}{$\begin{array}{l}\text { Overall } \\
\text { accuracy }\end{array}$} & Dice Similarity Coefficient (DSC) & $\frac{2 T P}{2 T P+F P+F N}$ & Similarity between model segmentation and reference segmentation. \\
\hline & Sensitivity & $\frac{T P}{T P+F N}$ & $\begin{array}{l}\text { Ratio of the overlapped segmentation to the reference segmentation, indicating how well } \\
\text { the model grasps the existing knowledge given by the reference. }\end{array}$ \\
\hline & Precision & $\frac{T P}{T P+F P}$ & $\begin{array}{l}\text { Ratio of the overlapped segmentation to the model segmentation, indicating the percentage } \\
\text { of segmented volume inferred by the model }\end{array}$ \\
\hline \multirow[t]{4}{*}{$\begin{array}{l}\text { Structural } \\
\text { accuracy }\end{array}$} & Branch Detected (BD) & $\frac{N_{T P}}{N_{\text {ref }}} \times 100 \%$ & $\begin{array}{l}\text { The number of branches in the overlapped segmentation over the number of branches in } \\
\text { the reference segmentation, indicating how well the model identifies the total number of } \\
\text { existing airways in the reference. }\end{array}$ \\
\hline & Tree-length Detected (TD) & $\frac{L_{T P}}{L_{\text {ref }}} \times 100 \%$ & $\begin{array}{l}\text { The length of airway tree in the overlapped segmentation over the length of airway tree } \\
\text { in the reference segmentation, indicating how well the model identifies the total length of } \\
\text { existing airways in the reference. }\end{array}$ \\
\hline & Branch Ratio $(\mathrm{BR})^{*}$ & $\frac{N_{P}}{N_{\text {ref }}} \times 100 \%$ & $\begin{array}{l}\text { The number of branches in the model segmentation over the number of branches in the } \\
\text { reference segmentation, indicating how well the model learns the airway features hidden } \\
\text { in the reference segmentation and uses these features to find more bronchioles. }\end{array}$ \\
\hline & Tree-length Ratio (TR) ${ }^{*}$ & $\frac{L_{P}}{L_{\text {ref }}} \times 100 \%$ & $\begin{array}{l}\text { The length of airway tree in the model segmentation over the length of airway tree in the } \\
\text { reference segmentation, indicating how well the model learns the airway features hidden } \\
\text { in the reference segmentation and uses these features to find more bronchioles. }\end{array}$ \\
\hline
\end{tabular}

${ }^{*}$ Note that the extra airways which do not exist in reference should be examined visually upon deletion of noises and exclusion of the influence from pulmonary vessels.

set (10 cases). Cross-validation was conducted by repeating the splitting multiple times, performing training, testing for each splitting, and taking the average of testing accuracy values. The remaining 20 cases (CASE21 to CASE40) from EXACT'09 were labeled with the same method by us (denoted as EXACT'09-TE). For the private dataset QMH, nine cases (denoted as QMH-L) of different slice thicknesses and pixel spacings (Table I) were labeled using commercial software named LungPoint with experts' manual correction.

The training set of EXACT'09-TR-LIDC-IDRI was used for supervised learning, while the remaining 978 unlabeled cases from LIDC-IDRI and 12 unlabeled cases from QMH were used for semi-supervised learning (denoted as LIDC-IDRIQMH-U). After training, the testing set of EXACT'09-TRLIDC-IDRI was used for internal testing, and QMH-L was used for external testing because the data distribution was unseen by the model.

\section{B. Implementation}

1) Data preparation: First, we stacked up the CT slices (in DICOM format) to form 3D image data. Then, thresholding was done to keep the Hounsfield Unit (HU) values within [$1000,600]$. Both the training sets of EXACT'09-TR-LIDCIDRI and LIDC-IDRI-QMH-U were further cropped into $32 \times 128 \times 128$ cuboids owing to available GPU memory.
2) Model development: We used PyTorch [46] to implement our model. Besides the techniques built by ourselves described in the Method section, data augmentation, including random flip, random affine, random blur, random noise, random motion, and random spike [44], was performed to further increase model robustness. We trained the model on an NVIDIA Tesla M60 with 8 GB memory for approximately three days. Adam optimizer and a learning rate of $10^{-5}$ were used during training.

\section{Metrics}

Ideally, the accuracy of model-based airway segmentation is best evaluated through actual bronchoscopy by experts. However, it is labor-intensive, and any additional examination that may prolong the clinical procedure should be avoided. For this pilot study on retrospective data, we devised seven metrics as listed in Table II for quantitative evaluation. These metrics can be divided into two categories-overall accuracy and structural accuracy. The former quantifies volumetric information voxel-wise, while the latter counts the number of branches and the length of detected tree structure. Three metrics are pertinent to overall accuracy and calculated from TP, FP, and FN, which denote true positive, false positive, and false negative, respectively. TP indicates percentage of model-based airway segmentation that overlaps with the reference airway segmentation; FP represents the rate of airway segments which 
only exist in model segmentation; FN signifies the proportion of reference airway segments that are not detected by model segmentation. These seven metrics constitute a more complete evaluation of the model.

In structural accuracy, we proposed two new metrics-Branch Ratio (BR) and Tree-length Ratio (TR) - to supplement Branch Detected (BD) and Tree-length Detected (TD), which were proposed by the EXACT'09 challenge and used in previous studies [8]. For BD and TD, the underlying assumption that FP segments are all noise is not necessarily valid. For deep learning models, FP segments may be airway branches missed by the reference annotation. Therefore, we introduced BR and TR. When calculating BR and TR, visual inspection was a preliminary step to check whether noises or pulmonary vessels were present in FP segmentation to verify that bronchioles to be included were real but overlooked by the reference.

In this study, we also calculated another widely used metric, False Positive Rate (FPR) but did not include it in Table $\Pi$ as FPR indicated the percentage of leakage volume. We argue that FPR may not be a good indicator for deep learningbased methods because it assumes all extra segments that do not exist in the reference are noise. Another concern is that FPR is defined as $\mathrm{FP} /(\mathrm{FP}+\mathrm{TN})$, in which $\mathrm{FP}+\mathrm{TN}$ stands for the background area, namely non-airways, in the reference segmentation. Different sizes of background areas were given in different published works [8], [29], [31], [33], [34], [47], making FPR an unstandardized metric for fair comparison. In our calculation, we only counted the background voxels within the cuboid, which was just fit by the airway reference mask in FP+TN. FPR was thus only used for our reference and found to be less reliable for model performance assessment than the other adopted metrics.

Additionally, we show the results of all the seven metrics of NaviAirway in Table VI in Results. However, when comparing NaviAirway with existing methods, not all the seven metrics were used because some accuracy results of other models were directly cited from literature. We did not reimplemented them because the source codes were not provided while they were trained and tested on the same datasets as ours. Moreover, we believe that they provided their best results, and our reimplementation would perform worse. Therefore, it is a fairer comparison by using their reported accuracy results.

\section{Results}

\section{A. Performance comparison}

First, our pipeline is compared with state-of-the-art airway segmentation methods on the testing sets of both EXACT'09TR-LIDC-IDRI and EXACT'09-TE. Table IV shows the comparison on the testing set of EXACT'09-TR-LIDC-IDRI. Our method outperformed others in both overall and structural accuracy. Note that we tested our method using two threshold values: 0.5 and 0.7 . Setting the threshold value to be 0.5 was to identify more bronchioles of higher generations, some of which were not included in the reference but were real airway segments by experts' visual inspection. Setting the threshold value to be 0.7 was to make a fair comparison in

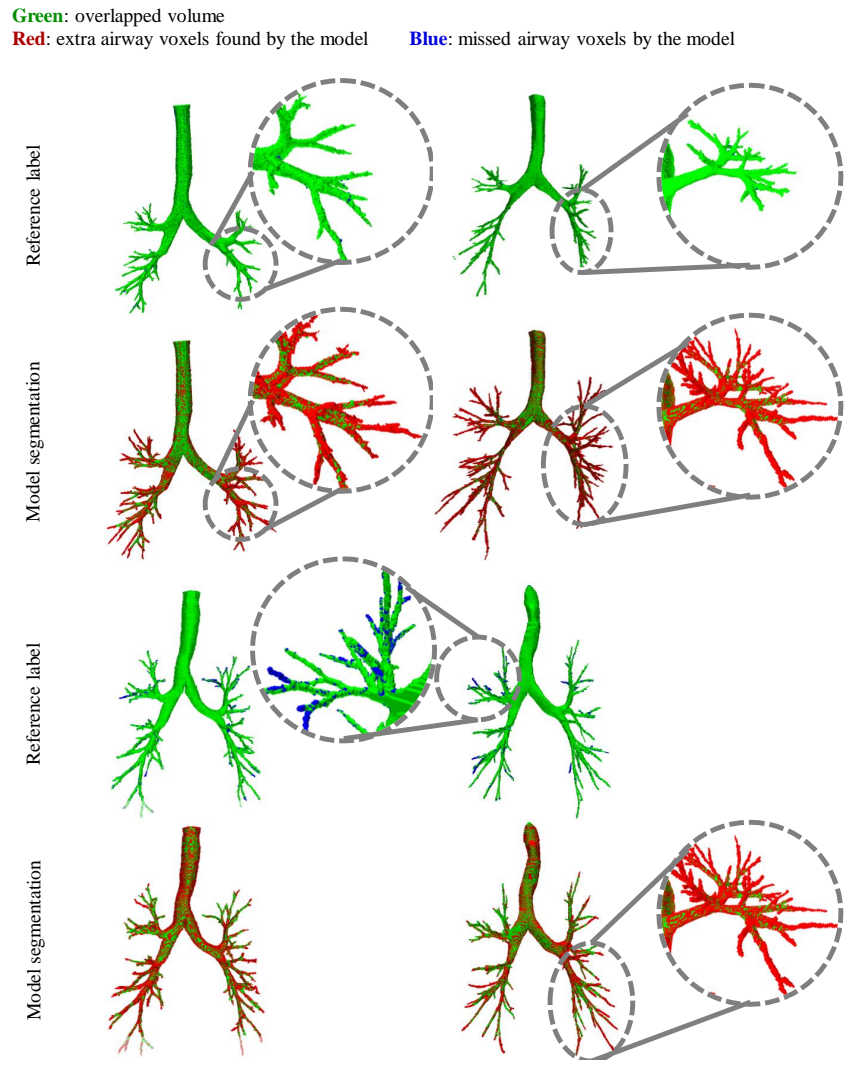

Fig. 3. Exemplary test results on EXACT'09-TR-LIDC-IDRI. (a) is a randomly chosen case. (b) and (c) are good cases where the model detected almost all the airways in the reference model. (d) is a case with relativity inferior performance and more undetected branches (the blue voxels) than others; nevertheless, the model only missed few airway branches and detected extra airways (the red voxels) overlooked by the reference.

TABLE III

THE VALUES OF THE SEVEN METRICS PLUS FPR OF (A)-(D) SHOWN IN FIGURE 3

\begin{tabular}{lllllllll}
\hline & DSC & Sensitivity & Precision & BD & TD & BR & TR & FPR \\
\hline (a) & $92.0 \%$ & $98.5 \%$ & $86.3 \%$ & $95.7 \%$ & $95.2 \%$ & $104.3 \%$ & $102.5 \%$ & $0.17 \%$ \\
(b) & $92.3 \%$ & $99.7 \%$ & $86.0 \%$ & $98.8 \%$ & $100.0 \%$ & $148.0 \%$ & $133.9 \%$ & $0.19 \%$ \\
(c) & $87.6 \%$ & $99.9 \%$ & $77.9 \%$ & $98.5 \%$ & $99.7 \%$ & $175.9 \%$ & $181.6 \%$ & $0.29 \%$ \\
(d) & $91.6 \%$ & $98.3 \%$ & $85.8 \%$ & $89.4 \%$ & $95.8 \%$ & $113.9 \%$ & $112.0 \%$ & $0.15 \%$ \\
\hline
\end{tabular}

terms of overall accuracy. From Table IV, our method had the highest overall accuracy with its DSC and sensitivity being respectively $90.7 \% \pm 1.8 \%$ and $98.4 \% \pm 1.4 \%$ at the threshold of 0.5 and $94.2 \% \pm 1.1 \%$ and $96.6 \% \pm 2.3 \%$ at the threshold of 0.7. In the aspect of structural accuracy, our method in the case of threshold $=0.5$ delineated much longer airway trees (TD: $94.2 \% \pm 5.7 \%$ ). When counting extra airway segments which were validated by an expert's visual inspection, our method could find more bronchioles of higher generations than the reference (Table IV] BR: $115.0 \% \pm 19.0 \%$ and TR: $115.5 \% \pm 18.5 \%$ when threshold $=0.5$ ). Moreover, our method detected finer bronchioles up to the 12th generation, whereas the mean and median values of the detected generation number in testing cases were 7.9 and 7.5 , respectively.

Table V summarized the performance of our (rows 12), published (rows 3-6), and newly submitted methods in 
TABLE IV

PERFORMANCE COMPARISON OF AIRWAY SEGMENTATION METHODS ON EXACT'09-TR-LIDC-IDRI.

THE MEAN VALUE AND STANDARD DEVIATION ARE SHOWN FOR EACH METRIC.

\begin{tabular}{|c|c|c|c|c|c|}
\hline & DSC & Sensitivity & $\mathrm{BD}$ & $\mathrm{TD}$ & FPR \\
\hline AG U-Net $[25]^{* *}$ & $82.7 \% \pm 22.2 \%$ & $72.5 \% \pm 28.9 \%$ & $70.1 \% \pm 33.3 \%$ & $63.5 \% \pm 30.8 \%$ & $0.014 \% \pm 0.012 \%$ * \\
\hline Wang et al. [26] & $93.5 \% \pm 2.2 \%$ & $88.6 \% \pm 8.8 \%$ & $93.4 \% \pm 8.0 \%$ & $85.6 \% \pm 9.9 \%$ & $0.018 \% \pm 0.012 \% *$ \\
\hline Juarez et al. [27] ${ }^{* *}$ & $87.5 \% \pm 13.2 \%$ & $77.5 \% \pm 15.5 \%$ & $77.5 \% \pm 20.9 \%$ & $66.0 \% \pm 20.4 \%$ & $0.009 \% \pm 0.009 \% *$ \\
\hline AirwayNet [30] & $93.7 \% \pm 1.9 \%$ & $87.2 \% \pm 8.9 \%$ & $91.6 \% \pm 8.3 \%$ & $82.1 \% \pm 10.9 \%$ & $0.014 \% \pm 0.009 \% *$ \\
\hline Juarez et al. [22] ${ }^{* *}$ & $93.6 \% \pm 2.2 \%$ & $86.7 \% \pm 9.1 \%$ & $91.9 \% \pm 9.2 \%$ & $80.7 \% \pm 11.3 \%$ & $0.014 \% \pm 0.009 \% *$ \\
\hline Jin et al. $[21]^{* *}$ & $93.6 \% \pm 2.0 \%$ & $88.1 \% \pm 8.5 \%$ & $93.1 \% \pm 7.9 \%$ & $84.8 \% \pm 9.9 \%$ & $0.017 \% \pm 0.010 \% *$ \\
\hline Qin et al. [33] ${ }^{* *}$ & $92.5 \% \pm 2.0 \%$ & $93.6 \% \pm 5.0 \%$ & $96.2 \% \pm 5.8 \%$ & $90.7 \% \pm 6.9 \%$ & $0.035 \% \pm 0.014 \% *$ \\
\hline 3D U-Net [20] ${ }^{* * * *}$ & $92.9 \% \pm 1.7 \%$ & $95.8 \% \pm 2.3 \%$ & $66.5 \% \pm 18.8 \%$ & $72.3 \% \pm 18.8 \%$ & $0.048 \% \pm 0.038 \%$ \\
\hline V-Net [48] $]^{* * * *}$ & $85.9 \% \pm 3.4 \%$ & $81.8 \% \pm 7.0 \%$ & $34.2 \% \pm 9.1 \%$ & $35.0 \% \pm 9.8 \%$ & $0.177 \% \pm 0.074 \%$ \\
\hline VoxResNet [49] ${ }^{* * *}$ & $85.8 \% \pm 6.3 \%$ & $78.3 \% \pm 9.8 \%$ & $29.8 \% \pm 9.9 \%$ & $33.1 \% \pm 10.2 \%$ & $0.044 \% \pm 0.023 \%$ \\
\hline Ours $(\text { th }=0.5)^{* * * *}$ & $90.7 \% \pm 1.8 \%$ & $98.4 \% \pm 1.4 \%$ & $88.4 \% \pm 10.7 \%$ & $94.2 \% \pm 5.7 \%$ & $0.224 \% \pm 0.128 \%$ \\
\hline Ours $(\text { th }=0.7)^{* * * *}$ & $94.2 \% \pm 1.1 \%$ & $96.6 \% \pm 2.3 \%$ & $83.3 \% \pm 11.4 \%$ & $90.4 \pm 8.4 \%$ & $0.117 \% \pm 0.079 \%$ \\
\hline
\end{tabular}

* The definition FPR of other methods may be different from ours as the exact computation of TN was not clarified.

** Rows 1-7 are state-of-the-art methods with performance values quoted from published articles (tested on same datasets as our method)

*** Rows 8-10 are three well-received medical image processing models reimplemented by us to do airway segmentation using dice loss function.

**** Rows 11 and 12 show the performance of our method when the thresholds are 0.5 and 0.7 , respectively.

TABLE V

PERformance COMPARISON OF AIRWAY SEGMENTATION METHODS ON EXACT'09-TE.

THE MEAN VALUE AND STANDARD DEVIATION ARE SHOWN FOR EACH METRIC.

\begin{tabular}{|c|c|c|c|c|c|}
\hline & Branch count & Tree length $(\mathrm{cm})$ & $\mathrm{BD} / \mathrm{BR}$ & TD/TR & FPR \\
\hline Ours (w/o semi) & $219.6 \pm 105.5$ & $243.2 \pm 127.6$ & (BR) $96.5 \% \pm 34.3 \%{ }^{*}$ & (TR) $114.7 \% \pm 40.1 \%^{*}$ & $0.072 \% \pm 0.021 \%$ \\
\hline Ours (w/ semi) & $272.9 \pm 142.4$ & $286.6 \pm 158.4$ & (BR) $117.7 \% \pm 38.0 \% *$ & (TR) $132.2 \% \pm 39.8 \%{ }^{*}$ & $0.094 \% \pm 0.025 \%$ \\
\hline $\mathrm{Xu}$ et al. [47] & $128.7 \pm 60.3$ & $94.8 \pm 44.7$ & $51.7 \% \pm 10.8 \%$ & $44.5 \% \pm 9.4 \%$ & $0.85 \% \pm 1.6 \%{ }^{* *}$ \\
\hline Yun et al. [29] & $163.4 \pm 79.4$ & $129.3 \pm 66.0$ & $65.7 \% \pm 13.1 \%$ & $60.1 \% \pm 11.9 \%$ & $4.5 \% \pm 3.7 \%{ }^{* *}$ \\
\hline Qin et al. [33] & $190.4^{* * *}$ & $166.5^{* * *}$ & $76.7 \% \pm 11.5 \%$ & $72.7 \% \pm 11.6 \%$ & $3.7 \% \pm 2.9 \%{ }^{* *}$ \\
\hline Zheng et al. [35] & $199.9^{* * * *}$ & $180.9^{* * *}$ & $80.5 \% \pm 12.5 \%$ & $79.0 \% \pm 11.1 \%$ & $5.8 \% \pm 4.3 \%{ }^{* *}$ \\
\hline Neko [38] & $84.5 \pm 40.5$ & $61.9 \pm 30.9$ & $35.5 \% \pm 8.2 \%$ & $30.4 \% \pm 7.4 \%$ & $0.89 \% \pm 1.8 \%{ }^{* *}$ \\
\hline UCCTeam [39] & $99.0 \pm 50.3$ & $75.1 \pm 39.4$ & $41.6 \% \pm 9.0 \%$ & $36.5 \% \pm 7.6 \%$ & $0.71 \% \pm 1.67 \%$ ** \\
\hline FF_ITC [40] & $198.3 \pm 98.6$ & $177.1 \pm 97.0$ & $79.6 \% \pm 13.5 \%$ & $79.9 \% \pm 12.1 \%$ & $11.92 \% \pm 13.16 \%{ }^{* *}$ \\
\hline MISLAB [8] & $104.7 \pm 55.2$ & $78.7 \pm 41.7$ & $42.9 \% \pm 9.6 \%$ & $37.5 \% \pm 7.1 \%$ & $0.89 \% \pm 1.64 \%^{* *}$ \\
\hline NTNU [50] & $72.4 \pm 37.8$ & $54.3 \pm 33.9$ & $31.3 \% \pm 10.4 \%$ & $27.4 \% \pm 9.6 \%$ & $3.60 \% \pm 3.37 \%{ }^{* *}$ \\
\hline
\end{tabular}

* We report the BR and TR values of our method and the BD and TD values of the compared methods.

** The calculation approach of these benchmark methods may be different from ours because their used TN was not specified.

*** Branch count and tree length values were not reported in [25] and [52]. We calculated them based on the reported BD and TD values.

TABLE VI

ABLATION STUDY AND MODEL PERFORMANCE ON THE PRIVATE DATASET (QMH). THE MEAN VALUE AND STANDARD DEVIATION ARE SHOWN FOR EACH METRIC. "SEMI" DENOTES SEMI-SUPERVISED LEARNING AND "DATA AUG" DENOTES DATA AUGMENTATION.

\begin{tabular}{|c|c|c|c|c|c|c|c|c|c|}
\hline $\mathrm{Th}$ & & DSC & Sensitivity & Precision & $\mathrm{BD}$ & $\mathrm{TD}$ & BR & TR & FPR \\
\hline \multirow[t]{6}{*}{0.5} & Ours & $90.7 \% \pm 1.8 \%$ & $98.4 \% \pm 1.4 \%$ & $84.2 \% \pm 3.4 \%$ & $88.4 \% \pm 10.7 \%$ & $94.2 \% \pm 5.7 \%$ & $115.0 \% \pm 19.0 \%$ & $115.5 \% \pm 18.5 \%$ & $0.224 \% \pm 0.128 \%$ \\
\hline & w/o dilated conv + self-attention & $90.5 \% \pm 1.5 \%$ & $97.3 \% \pm 2.2 \%$ & $84.8 \% \pm 3.1 \%$ & $81.0 \% \pm 12.2 \%$ & $85.5 \% \pm 10.5 \%$ & $103.3 \% \pm 28.7 \%$ & $99.4 \% \pm 24.3 \%$ & $0.201 \% \pm 0.115 \%$ \\
\hline & $\mathrm{w} /$ org dice & $93.9 \% \pm 1.3 \%$ & $93.5 \% \pm 3.1 \%$ & $94.4 \% \pm 1.9 \%$ & $71.3 \% \pm 14.1 \%$ & $74.0 \% \pm 14.1 \%$ & $73.1 \% \pm 19.3 \%$ & $76.9 \% \pm 19.4 \%$ & $0.065 \% \pm 0.046 \%$ \\
\hline & w/o iterative training & $90.7 \% \pm 1.3 \%$ & $95.7 \% \pm 3.0 \%$ & $86.4 \% \pm 2.9 \%$ & $76.1 \% \pm 14.8 \%$ & $80.4 \% \pm 13.4 \%$ & $90.6 \% \pm 23.7 \%$ & $90.5 \% \pm 23.0 \%$ & $0.180 \% \pm 0.109 \%$ \\
\hline & w/o semi & $91.6 \% \pm 1.5 \%$ & $97.7 \% \pm 1.6 \%$ & $86.4 \% \pm 3.1 \%$ & $83.7 \% \pm 11.2 \%$ & $89.8 \% \pm 7.8 \%$ & $104.2 \% \pm 26.8 \%$ & $106.4 \% \pm 25.4 \%$ & $0.180 \% \pm 0.116 \%$ \\
\hline & w/o data aug & $93.2 \% \pm 1.4 \%$ & $97.7 \% \pm 1.7 \%$ & $89.1 \% \pm 3.2 \%$ & $86.9 \% \pm 9.8 \%$ & $89.3 \% \pm 8.7 \%$ & $112.9 \% \pm 32.8 \%$ & $107.8 \% \pm 29.2 \%$ & $0.135 \% \pm 0.071 \%$ \\
\hline \multirow[t]{6}{*}{0.7} & Ours & $94.2 \% \pm 1.1 \%$ & $96.6 \% \pm 2.3 \%$ & $91.2 \% \pm 2.6 \%$ & $83.3 \% \pm 11.4 \%$ & $90.4 \pm 8.4 \%$ & $93.6 \% \pm 22.6 \%$ & $100.7 \% \pm 23.6 \%$ & $0.117 \% \pm 0.079 \%$ \\
\hline & w/o dilated conv + self-attention & $93.1 \% \pm 1.2 \%$ & $95.3 \% \pm 3.1 \%$ & $91.0 \% \pm 2.4 \%$ & $74.8 \% \pm 13.7 \%$ & $79.9 \% \pm 12.6 \%$ & $80.8 \% \pm 19.2 \%$ & $86.3 \% \pm 20.1 \%$ & $0.110 \% \pm 0.071 \%$ \\
\hline & $\mathrm{w} /$ org dice & $93.4 \% \pm 1.8 \%$ & $90.2 \% \pm 3.9 \%$ & $96.9 \% \pm 1.3 \%$ & $61.8 \% \pm 14.8 \%$ & $70.0 \% \pm 17.7 \%$ & $62.2 \% \pm 14.1 \%$ & $69.0 \% \pm 14.9 \%$ & $0.035 \% \pm 0.025 \%$ \\
\hline & w/o iterative training & $92.6 \% \pm 1.5 \%$ & $93.2 \% \pm 3.9 \%$ & $92.1 \% \pm 2.1 \%$ & $70.8 \% \pm 16.2 \%$ & $76.3 \% \pm 14.2 \%$ & $78.6 \% \pm 21.4 \%$ & $81.5 \% \pm 19.4 \%$ & $0.094 \% \pm 0.064 \%$ \\
\hline & w/o semi & $93.9 \% \pm 2.4 \%$ & $95.3 \% \pm 2.8 \%$ & $92.7 \% \pm 2.3 \%$ & $76.6 \% \pm 11.9 \%$ & $82.7 \% \pm 11.0 \%$ & $86.9 \% \pm 21.9 \%$ & $91.8 \% \pm 22.2 \%$ & $0.090 \% \pm 0.068 \%$ \\
\hline & w/o data aug & $94.8 \% \pm 0.9 \%$ & $95.5 \% \pm 2.4 \%$ & $94.4 \% \pm 2.5 \%$ & $80.1 \% \pm 10.6 \%$ & $84.8 \% \pm 9.9 \%$ & $92.9 \% \pm 23.2 \%$ & $95.0 \% \pm 23.0 \%$ & $0.066 \% \pm 0.046 \%$ \\
\hline \multirow[t]{2}{*}{0.5} & QMH & 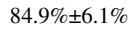 & 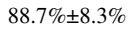 & 87 & 170 & 12 & 10 & $88.2 \% \pm 9.7 \%$ & 0.03 \\
\hline & QMH w/ semi & $85.5 \% \pm 4.7 \%$ & $95.4 \% \pm 1.5 \%$ & $77.8 \% \pm 8.1 \%$ & $90.2 \% \pm 7.8 \%$ & $83.7 \% \pm 6.2 \%$ & $139.7 \% \pm 23.7 \%$ & $106.0 \% \pm 14.5 \%$ & $0.058 \% \pm 0.045 \%$ \\
\hline
\end{tabular}

the EXACT'09 challenge (rows 7-11) on the EXACT'09TE dataset. Setting the threshold to be 0.5 , we considered our method in two conditions: with semi-supervised learning (i.e., Ours (w/ semi) in Table V and without semi-supervised learning (i.e., Ours (w/o semi) in Table V). Because the reference labels of EXACT'09-TE are not publicly available and the methods used for comparison (from Row 2 to Row 9) in Table $\mathrm{V}$ may have used different reference labels, to have a fair comparison, we used branch count and tree length $(\mathrm{cm})$ values as they were more reference-independent, 
TABLE VII

BRANCH COUNT, TREE LENGTH, BR, TR, AND DETECTED AIRWAY GENERATIONS FOR THE 20 CASES IN EXACT'09-TE. OUR METHOD WAS DEVELOPED WITH AND WITHOUT SEMI-SUPERVISED LEARNING.

\begin{tabular}{|c|c|c|c|c|c|c|c|c|c|c|c|c|c|}
\hline & \multicolumn{3}{|c|}{ Branch count } & \multirow{2}{*}{$\begin{array}{l}\text { BR } \\
\text { (w/o semi) }\end{array}$} & \multirow{2}{*}{$\begin{array}{l}\text { BR } \\
\text { (w/ semi) }\end{array}$} & \multicolumn{3}{|c|}{ Tree length $(\mathrm{cm})$} & \multirow{2}{*}{$\begin{array}{l}\text { TR } \\
\text { (w/o semi) }\end{array}$} & \multirow{2}{*}{$\begin{array}{l}\text { TR } \\
\text { (w/ semi) }\end{array}$} & \multicolumn{3}{|c|}{ Airway generation (w/ semi) } \\
\hline & $\operatorname{Ref}^{*}$ & w/o semi & w/ semi & & & $\operatorname{Ref}^{*}$ & w/o semi & w/ semi & & & Avg & Median & $\operatorname{Max}$ \\
\hline CASE21 & 200 & 154 & 174 & $77.0 \%$ & $87.0 \%$ & 120.3 & 135.1 & 155.3 & $112.3 \%$ & $129.2 \%$ & 5.7 & 6 & 12 \\
\hline CASE22 & 388 & 271 & 328 & $69.8 \%$ & $84.5 \%$ & 341.5 & 296 & 357.6 & $86.7 \%$ & $104.7 \%$ & 8.3 & 9 & 17 \\
\hline CASE23 & 285 & 123 & 275 & $43.2 \%$ & $96.5 \%$ & 271.3 & 154.5 & 361.3 & $57.0 \%$ & $133.2 \%$ & 7.2 & 7 & 15 \\
\hline CASE24 & 187 & 267 & 319 & $142.8 \%$ & $170.6 \%$ & 185.8 & 339.4 & 377 & $182.7 \%$ & $202.9 \%$ & 5.8 & 7 & 15 \\
\hline CASE25 & 235 & 365 & 371 & $155.3 \%$ & $157.9 \%$ & 270.2 & 451.4 & 437.7 & $167.1 \%$ & $162.0 \%$ & 4.5 & 7 & 15 \\
\hline CASE26 & 81 & 107 & 111 & $132.1 \%$ & $137.0 \%$ & 83.2 & 142.6 & 128.9 & $171.5 \%$ & $155.0 \%$ & 4.7 & 5 & 9 \\
\hline CASE27 & 102 & 115 & 151 & $112.7 \%$ & $148.0 \%$ & 94.4 & 139.7 & 171.7 & $148.0 \%$ & $182.0 \%$ & 4.9 & 5 & 10 \\
\hline CASE28 & 124 & 167 & 187 & $134.7 \%$ & $150.8 \%$ & 122 & 201.1 & 227.5 & $164.7 \%$ & $186.4 \%$ & 7 & 7 & 13 \\
\hline CASE29 & 185 & 191 & 211 & $103.2 \%$ & $114.1 \%$ & 150.3 & 227.5 & 237.9 & $151.4 \%$ & $158.3 \%$ & 7.4 & 8 & 13 \\
\hline CASE30 & 196 & 177 & 211 & $90.3 \%$ & $107.7 \%$ & 164.4 & 178.4 & 187.5 & $108.6 \%$ & $114.1 \%$ & 5.7 & 6 & 12 \\
\hline CASE31 & 215 & 175 & 191 & $81.4 \%$ & $88.8 \%$ & 188.4 & 187.1 & 195.3 & $99.3 \%$ & $103.7 \%$ & 5.9 & 6 & 12 \\
\hline CASE32 & 234 & 156 & 174 & $66.7 \%$ & $74.4 \%$ & 231.4 & 199.7 & 212.9 & $86.3 \%$ & $92.0 \%$ & 6.3 & 7 & 11 \\
\hline CASE33 & 169 & 187 & 220 & $110.7 \%$ & $130.2 \%$ & 159.6 & 203.5 & 230.9 & $127.5 \%$ & $144.7 \%$ & 6.1 & 6 & 12 \\
\hline CASE34 & 459 & 324 & 442 & $70.6 \%$ & $96.3 \%$ & 368.8 & 361.5 & 440.7 & $98.0 \%$ & $119.5 \%$ & 7.9 & 8 & 17 \\
\hline CASE35 & 345 & 302 & 335 & $87.5 \%$ & $97.1 \%$ & 320.4 & 286.4 & 305.5 & $89.4 \%$ & $95.3 \%$ & 6.6 & 7 & 12 \\
\hline CASE36 & 365 & 525 & 741 & $143.8 \%$ & $203.0 \%$ & 422.6 & 615.6 & 792.9 & $145.7 \%$ & $187.6 \%$ & 8.7 & 9 & 18 \\
\hline CASE37 & 186 & 237 & 291 & $127.4 \%$ & $156.5 \%$ & 190.3 & 115.9 & 135.1 & $60.9 \%$ & $71.0 \%$ & 6.8 & 7 & 15 \\
\hline CASE38 & 99 & 78 & 121 & $78.8 \%$ & $122.2 \%$ & 78.2 & 81.6 & 108.9 & $104.4 \%$ & $139.4 \%$ & 4.2 & 5 & 10 \\
\hline CASE39 & 521 & 279 & 369 & $53.6 \%$ & $70.8 \%$ & 420.1 & 306.5 & 395.6 & $73.0 \%$ & $94.2 \%$ & 8.6 & 9 & 15 \\
\hline CASE40 & 390 & 191 & 236 & $49.0 \%$ & $60.5 \%$ & 399.3 & 239.6 & 271.7 & $60.0 \%$ & $68.0 \%$ & 6.7 & 7 & 13 \\
\hline$\overline{\text { Avg }}$ & 248.3 & 219.6 & 272.9 & $96.5 \%$ & $117.7 \%$ & 229.1 & 243.2 & 286.6 & $114.7 \%$ & $132.2 \%$ & 6.5 & 6.9 & 13.3 \\
\hline Std & 124.5 & 105.5 & 142.4 & $34.3 \%$ & $38.0 \%$ & 115.3 & 127.6 & 158.4 & $40.1 \%$ & $39.8 \%$ & 1.3 & 1.3 & 2.5 \\
\hline Max & 521 & 525 & 741 & $155.3 \%$ & $203.0 \%$ & 422.6 & 615.6 & 792.9 & $182.7 \%$ & $202.9 \%$ & 8.7 & 9 & 18 \\
\hline 1st quartile & 181 & 156 & 184 & $70.4 \%$ & $88.4 \%$ & 143.2 & 151.6 & 183.6 & $86.6 \%$ & $101.6 \%$ & 5.7 & 6 & 12 \\
\hline Median & 208 & 189 & 228 & $88.9 \%$ & $110.9 \%$ & 189.4 & 202.3 & 234.4 & $106.5 \%$ & $131.2 \%$ & 6.5 & 7 & 13 \\
\hline 3rd quartile & 350 & 273 & 330 & $128.6 \%$ & $148.7 \%$ & 325.6 & 298.6 & 365.2 & $148.9 \%$ & $159.2 \%$ & 7.2 & 7.3 & 15 \\
\hline MIN & 81 & 78 & 111 & $43.2 \%$ & $60.5 \%$ & 78.2 & 81.6 & 108.9 & $57.0 \%$ & $68.0 \%$ & 4.2 & 5 & 9 \\
\hline
\end{tabular}

* The "Ref" columns list the corresponding values of the reference labels quoted from the website of the EXACT'09 challenge.
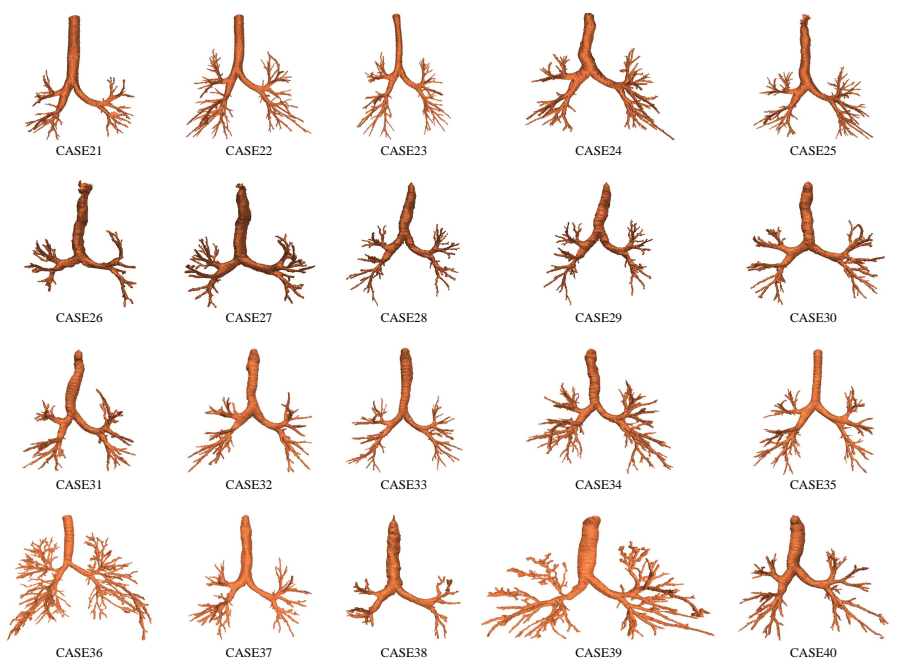

Fig. 4. Exemplary segmentation results obtained by our method with semi-supervised learning on EXACT'09-TE.

especially when the FPR was small. Results showed that our method without semi-supervised learning already detected two times more branches and two times longer airway trees than benchmarks. With semi-supervised learning, our method performed even better (Branch count: 272.9 \pm 142.4 and Tree length (cm): 286.6 \pm 158.4$)$.

Overall, from the two comparisons on EXACT'09-TRLIDC-IDRI and EXACT'09-TE, we observed that our model had higher overall accuracy and detected longer airway branches. Particularly, high sensitivity indicates that our method found almost all the airway segments in the reference, while high BR and TR (more than 100\%) mean that our method detected some airway segments overlooked by the reference.

Figure 4 shows airways of 20 cases in EXACT'09-TE segmented by our method with semi-supervised learning. Table VII summarized the detailed branch count, tree length values, and the airway generations detected by our method with and without semi-supervised learning for each of the 20 cases. The reported values of reference labels were taken from the website of EXACT'09 challenge, provided in the "Ref" columns, and used to calculate the BR and TR values. Results showed that our method could detect more bronchioles (BR: $117.7 \%$ and TR: $132.2 \%$ ) of higher generations, up to the 13th generation on average. For CASE36, our model detected twice more branches and twice longer airway trees, and the detected bronchioles were up to the 18th generation.

\section{B. Ablation study}

We tested how each of the components in our model contributed to performance improvement. Table VI shows that all components improved segmentation performance (e.g., around $10 \%$ to $30 \%$ improvement in terms of TR), while the data augmentation strategy only slightly improved model performance in terms of Sensitivity, BD, TD, BR, and TR.

1) Effect of the dilated convolution plus self-attention modules.: The "w/o dilated conv + self-attention" rows in Table VI showed equally good overall accuracy as our method with "dilated conv+self-attention" but significantly lower structural accuracy in both thresholding conditions, e.g., TD from $94.2 \%$ to $85.5 \%$ and TR from $115.5 \%$ to $99.4 \%$ at the threshold 
of 0.5 . This demonstrated that the added modules improved model performance by a considerable margin.

2) Effect of the proposed loss function.: Comparing the use of our new loss function with the original dice loss function ("w/ org dice" rows in Table VI), we noted a significant increase in sensitivity (93.5\% to $98.4 \%$ ) but a decrease in precision ( $94.4 \%$ to $84.2 \%$ ) because the proposed loss function tends to identify airway branches missed in reference labels in our experiments.

3) Effect of iterative training.: In the ablation experiment, we replaced the iterative training strategy with same-frequency training, where all pre-cropped cuboids were selected with the same probability in each training batch. When using iterative training, the model improved on most metrics, particularly structural accuracy.

4) Effect of semi-supervised learning.: The performance comparison between our method with and without semisupervised learning was summarized in Tables IV, III and $\mathrm{V}$ With teacher-student training, our model learned more general features of airways and detected more bronchioles of high generations.

5) Effect of data augmentation.: Data augmentation was also attempted to increase model robustness. Improvement in metrics, except for DSC and precision, was noted in Table VI but not drastic enough.

\section{Test results on unseen images}

The bottom two rows in Table VI examined the performance of our model in the unseen dataset (QMH) (see section 3.1). Although model performance dropped when compared with that on the EXACT'09-TR-LIDC-IDRI dataset, our model still achieved high accuracy (DSC: $84.9 \%$ and $85.5 \%$, sensitivity: $88.7 \%$ and $95.4 \%$, where the accuracy values show the model performance without and with semi-supervised learning, respectively). In terms of structural accuracy, not only did average BD (from $75.6 \%$ to $90.2 \%$ ) and TD (from $72.9 \%$ to $83.7 \%$ ) increase but the standard deviations also became lower. This suggested that semi-supervised learning enhanced model robustness across different cases. Furthermore, with semisupervised learning, our method detected more bronchioles than the reference labels (BR: $139.7 \%$ and TR: $106.0 \%$ ).

\section{DISCUSSION}

1) A more comprehensive design is needed.: The performance improvement of our method was attributed to our more comprehensive and airway morphology-specific design strategy, which focused on not only the novel architecture of the deep learning model but also the loss function design, training strategy, and semi-supervised learning with unlabeled images. Besides, our method extended the model-centric view, in which many methods strived for model architecture design, and provided other options for airway segmentation performance improvement.

2) Be more data-centric.: One special challenge for medical image processing is the high labeling cost, which leads to a small number of labeled samples and a large amount of unlabeled data. An effective approach to using both groups of data, instead of only relying on labeled samples, shall improve model performance. We developed a semi-supervised training approach via teacher-student training and demonstrated that it significantly improved the model performance and robustness.

3) Training strategy matters.: From Table VI the effects of the proposed loss function and iterative training strategy were much more significant than other components. Unlike most earlier methods that followed a first-low-generation-thenhigh-generation training strategy, this study investigated the influence of different training strategies. In our settings, the first-coarse-then-fine training strategy performed a little bit poorer than training on all pre-cropped cuboids with the same frequency (Table VIII). This might be because training with a more focus on finer bronchioles in the second stage resulted in the loss of learned features of low-generation airways. On the other hand, first-fine-then-low-coarse training did not have satisfactory performance, either, because the extracted features of finer bronchioles might be lost when training on low generations. In contrast, our proposed iterative training could teach the model to learn unique features of both low and high airway generations while preserving the knowledge of airway features of both high and low generations.

It was noticed that, when too much focus was put on finer bronchioles, the performance dropped drastically to 0 at $\beta=$ 10. This was because the model would take the shortcut by just regarding all voxels as background when most images fed to the model only had a few airway voxels.

TABLE VIII

DSC VALUES UNDER DIFFERENT TRAINING STRATEGIES INCORPORATED INTO OUR PIPELINE (THRESHOLD $=0.7$ ).

\begin{tabular}{|c|c|c|c|}
\hline Strategy & DSC & Strategy & DSC \\
\hline More on coarse $(\beta=1)$ & $89.60 \%$ & First coarse then fine & $90.00 \%$ \\
\hline More on coarse $(\beta=10)$ & $90.40 \%$ & First fine then coarse & $88.20 \%$ \\
\hline More on fine $(\beta=1)$ & $87.30 \%$ & Same frequency & $92.60 \%$ \\
\hline More on fine $(\beta=10)$ & $\sim 0 \%$ & Iterative & $94.20 \%$ \\
\hline
\end{tabular}

4) The proposed bronchiole-sensitive loss function is more effective than the original dice loss function.: As shown in Table VI] in terms of sensitivity and structural accuracy, the proposed loss function performed significantly better (around $5 \%$ to $30 \%$ improvement) than the original dice loss function. The reason DSC and precision were higher when using the original dice loss function was that the original dice loss was more "conservative" than the proposed one and tended to circumscribe the airway segmentation within the airway mask in reference labels. Therefore, dice loss was deemed suboptimal because the labels used in this study served as a reference, not the ground truth.

5) Select the model of appropriate size.: Compared with VNet and VoxResNet in Table IV] 3D U-Net (size: $38 \mathrm{MB}$ ) was found to be more suitable for airway segmentation tasks. V-Net (size: $182 \mathrm{MB}$ ) might be too large, so it has a slow inference speed and is difficult to train; VoxResNet (size: $25 \mathrm{MB}$ ) might be too small and cannot process the level of complexity of chest CT images). That is why we implemented NaviAirway based on the structure of 3D U-Net. 
6) Review metrics for airway segmentation.: Most existing works followed the metrics built in the EXACT'09 challenge in 2009 [8]. However, because we only had the "reference" labels, instead of the "ground truth" segmentation, and give extensive applications of deep learning in recent years, we proposed to devise new metrics. First, quantitative evaluation was insufficient. We proposed to include visual inspection because the model segmentation might make mistakes in some image regions while finding more bronchioles than the reference in some others. Then, for quantitative metrics, we need to reconsider the implication of False Positive FP defined in Table II (also "leakage" in [8]). For traditional methods using techniques, such as region growing, FP may usually be meaningless leaking volumes. However, for deep learning approaches, after removing unconnected shapes, the FP may include the undetected airway segments in the reference labels. Therefore, both Branch Detected (BD) and Tree-length Detected (TD) metrics are not deemed comprehensive measures. As a result, we proposed Branch Ratio (BR) and Tree-length Ratio (TR) to compare the total number of branches and length of airway trees between model segmentation and reference labels. Moreover, False Positive Rate (FPR) may not be a good measure because it assumes all FP are meaningless leakage volumes and its definition is ambiguous.

7) Future directions.: To translate our method into clinical practice, further investigations are needed.

- Real-time Bayesian learning and human-computer interaction: The operation of navigational bronchoscopy is a proving-and-adjusting process. An ultrathin bronchoscope is inserted into a patient's airway following a preplanned airway roadmap. However, the roadmap may be wrong at a certain site in the planned pathway. For instance, at a certain generation, there may be three branches ahead shown in the real-time endoscopic image but only two branches in the provided roadmap are derived from segmentation. Real-time Bayesian learning may help resolve this situation through adjustment of airway segmentation prediction based on the observed airways by navigational bronchoscopy and subsequent feedback to the operator on the path with the highest probability to reach the target nodule as near as possible.

- Design of improved model robustness to tackle the problem of domain shift, i.e., training on some CT scans but application on another set of CT scans: It is not feasible to ask the clinicians to label the airways and retrain the model on the CT scans acquired at their affiliated hospitals. We need to build techniques to increase model robustness by making the model learn the features of airways in general CT scans, not a specific set of CT scans.

- Model interpretability to increase its trustworthiness to humans: Clinical applications are high-stakes scenarios. Humans naturally need explanations on a machine's decisions to use the machine wisely and give timely feedback on parameter settings and machine reconfiguration.

\section{CONCLUSIONS}

In this paper, we presented a novel airway segmentation pipeline to identify finer bronchioles, including feature extractor modules in model architecture design, a more effective loss function, and a human-behavior-inspired training strategy. The model knowledge became better preserved, the model could recognize finer bronchioles, and the unlabeled data could now be utilized by semi-supervised learning to increase model accuracy and model robustness. We tested our model on both public and private datasets. The results demonstrated that our pipeline was robust on new CT scans and outperformed existing methods. Our pipeline is envisioned to provide extensive airway roadmaps with more detailed bronchioles, showing more paths for pulmonary nodule sampling in navigation bronchoscopy. Additionally, the proposed loss function, the iterative training strategy, and the teacher-student training with morphology-specific data augmentation can also be applied to other scenarios when segmenting fine and long tubular structures in biomedical images.

\section{REFERENCES}

[1] T. Ishiwata, A. Gregor, T. Inage, and K. Yasufuku, "Bronchoscopic navigation and tissue diagnosis," General thoracic and cardiovascular surgery, vol. 68, no. 7, pp. 672-678, 2020.

[2] E. Edell and D. Krier-Morrow, "Navigational bronchoscopy: Overview of technology and practical considerations-new current procedural terminology codes effective 2010," Chest, vol. 137, no. 2, pp. 450-454, 2010.

[3] F. Asano, R. Eberhardt, and F. J. Herth, "Virtual bronchoscopic navigation for peripheral pulmonary lesions," Respiration, vol. 88, no. 5, pp. 430-440, 2014

[4] S. V. Kemp, "Navigation bronchoscopy," Respiration, vol. 99, no. 4, pp. 277-286, 2020.

[5] W. O. Reece, "Overview of the respiratory system," Dukes' physiology of domestic animals, vol. 203, 2015.

[6] E. M. Van Rikxoort and B. Van Ginneken, "Automated segmentation of pulmonary structures in thoracic computed tomography scans: a review," Physics in Medicine \& Biology, vol. 58, no. 17, p. R187, 2013.

[7] J. Pu, S. Gu, S. Liu, S. Zhu, D. Wilson, J. M. Siegfried, and D. Gur, "Ct based computerized identification and analysis of human airways: a review," Medical physics, vol. 39, no. 5, pp. 2603-2616, 2012.

[8] P. Lo, B. Van Ginneken, J. M. Reinhardt, T. Yavarna, P. A. De Jong, B. Irving, C. Fetita, M. Ortner, R. Pinho, J. Sijbers et al., "Extraction of airways from ct (exact'09)," IEEE Transactions on Medical Imaging, vol. 31, no. 11, pp. 2093-2107, 2012.

[9] D. Aykac, E. A. Hoffman, G. McLennan, and J. M. Reinhardt, "Segmentation and analysis of the human airway tree from three-dimensional x-ray ct images," IEEE transactions on medical imaging, vol. 22, no. 8, pp. 940-950, 2003.

[10] H. Shi, W. C. Scarfe, and A. G. Farman, "Upper airway segmentation and dimensions estimation from cone-beam ct image datasets," International Journal of Computer Assisted Radiology and Surgery, vol. 1, no. 3, pp. 177-186, 2006.

[11] I. Cheng, S. Nilufar, C. Flores-Mir, and A. Basu, "Airway segmentation and measurement in ct images," in 2007 29th Annual International Conference of the IEEE Engineering in Medicine and Biology Society. IEEE, 2007, pp. 795-799.

[12] J. Tschirren, E. A. Hoffman, G. McLennan, and M. Sonka, "Intrathoracic airway trees: segmentation and airway morphology analysis from lowdose ct scans," IEEE transactions on medical imaging, vol. 24, no. 12, pp. 1529-1539, 2005.

[13] _ "Segmentation and quantitative analysis of intrathoracic airway trees from computed tomography images," Proceedings of the American Thoracic Society, vol. 2, no. 6, pp. 484-487, 2005.

[14] A. Fabijańska, "Two-pass region growing algorithm for segmenting airway tree from mdct chest scans," Computerized Medical Imaging and Graphics, vol. 33, no. 7, pp. 537-546, 2009. 
[15] M. W. Graham, J. D. Gibbs, D. C. Cornish, and W. E. Higgins, "Robust 3-d airway tree segmentation for image-guided peripheral bronchoscopy," IEEE transactions on medical imaging, vol. 29, no. 4, pp. 982-997, 2010.

[16] C. Fetita, M. Ortner, P.-Y. Brillet, F. Prêteux, P. Grenier et al., "A morphological-aggregative approach for $3 \mathrm{~d}$ segmentation of pulmonary airways from generic msct acquisitions," in Proc. of Second International Workshop on Pulmonary Image Analysis, 2009, pp. 215-226.

[17] A. P. Kiraly, W. E. Higgins, G. McLennan, E. A. Hoffman, and J. M. Reinhardt, "Three-dimensional human airway segmentation methods for clinical virtual bronchoscopy," Academic radiology, vol. 9, no. 10, pp. 1153-1168, 2002.

[18] Q. Meng, T. Kitasaka, Y. Nimura, M. Oda, J. Ueno, and K. Mori, "Automatic segmentation of airway tree based on local intensity filter and machine learning technique in $3 \mathrm{~d}$ chest ct volume," International journal of computer assisted radiology and surgery, vol. 12, no. 2, pp. 245-261, 2017.

[19] B. van Ginneken, W. Baggerman, and E. M. van Rikxoort, "Robust segmentation and anatomical labeling of the airway tree from thoracic ct scans," in International Conference on Medical Image Computing and Computer-Assisted Intervention. Springer, 2008, pp. 219-226.

[20] Ö. Çiçek, A. Abdulkadir, S. S. Lienkamp, T. Brox, and O. Ronneberger, "3d u-net: learning dense volumetric segmentation from sparse annotation," in International conference on medical image computing and computer-assisted intervention. Springer, 2016, pp. 424-432.

[21] D. Jin, Z. Xu, A. P. Harrison, K. George, and D. J. Mollura, "3d convolutional neural networks with graph refinement for airway segmentation using incomplete data labels," in International workshop on machine learning in medical imaging. Springer, 2017, pp. 141-149.

[22] A. G.-U. Juarez, H. A. Tiddens, and M. de Bruijne, "Automatic airway segmentation in chest ct using convolutional neural networks," in Image analysis for moving organ, breast, and thoracic images. Springer, 2018, pp. 238-250.

[23] S. A. Nadeem, E. A. Hoffman, J. C. Sieren, A. P. Comellas, S. P. Bhatt, I. Z. Barjaktarevic, F. Abtin, and P. K. Saha, "A ct-based automated algorithm for airway segmentation using freeze-and-grow propagation and deep learning," IEEE Transactions on Medical Imaging, vol. 40, no. 1, pp. 405-418, 2020.

[24] A. Garcia-Uceda, R. Selvan, Z. Saghir, H. Tiddens, and M. de Bruijne, "Automatic airway segmentation from computed tomography using robust and efficient 3-d convolutional neural networks," arXiv preprint arXiv:2103.16328, 2021.

[25] J. Schlemper, O. Oktay, M. Schaap, M. Heinrich, B. Kainz, B. Glocker, and D. Rueckert, "Attention gated networks: Learning to leverage salient regions in medical images," Medical image analysis, vol. 53, pp. 197207, 2019

[26] C. Wang, Y. Hayashi, M. Oda, H. Itoh, T. Kitasaka, A. F. Frangi, and K. Mori, "Tubular structure segmentation using spatial fully connected network with radial distance loss for $3 \mathrm{~d}$ medical images," in International Conference on Medical Image Computing and Computer-Assisted Intervention. Springer, 2019, pp. 348-356.

[27] A. G.-U. Juarez, R. Selvan, Z. Saghir, and M. de Bruijne, "A joint 3d unet-graph neural network-based method for airway segmentation from chest cts," in International workshop on machine learning in medical imaging. Springer, 2019, pp. 583-591.

[28] R. Selvan, T. Kipf, M. Welling, A. G.-U. Juarez, J. H. Pedersen, J. Petersen, and M. de Bruijne, "Graph refinement based airway extraction using mean-field networks and graph neural networks," Medical Image Analysis, vol. 64, p. 101751, 2020.

[29] J. Yun, J. Park, D. Yu, J. Yi, M. Lee, H. J. Park, J.-G. Lee, J. B. Seo, and N. Kim, "Improvement of fully automated airway segmentation on volumetric computed tomographic images using a 2.5 dimensional convolutional neural net," Medical image analysis, vol. 51, pp. 13-20, 2019.

[30] Y. Qin, M. Chen, H. Zheng, Y. Gu, M. Shen, J. Yang, X. Huang, Y.-M. Zhu, and G.-Z. Yang, "Airwaynet: a voxel-connectivity aware approach for accurate airway segmentation using convolutional neural networks," in International Conference on Medical Image Computing and Computer-Assisted Intervention. Springer, 2019, pp. 212-220.

[31] Y. Qin, Y. Gu, H. Zheng, M. Chen, J. Yang, and Y.-M. Zhu, "Airwaynetse: A simple-yet-effective approach to improve airway segmentation using context scale fusion," in 2020 IEEE 17th International Symposium on Biomedical Imaging (ISBI). IEEE, 2020, pp. 809-813.

[32] H. Zhang, M. Shen, P. L. Shah, and G.-Z. Yang, "Pathological airway segmentation with cascaded neural networks for bronchoscopic navigation," in 2020 IEEE International Conference on Robotics and Automation (ICRA). IEEE, 2020, pp. 9974-9980.
[33] Y. Qin, H. Zheng, Y. Gu, X. Huang, J. Yang, L. Wang, F. Yao, Y.-M. Zhu, and G.-Z. Yang, "Learning tubule-sensitive cnns for pulmonary airway and artery-vein segmentation in ct," IEEE Transactions on Medical Imaging, vol. 40, no. 6, pp. 1603-1617, 2021.

[34] Y. Qin, H. Zheng, Y. Gu, X. Huang, J. Yang, L. Wang, and Y.M. Zhu, "Learning bronchiole-sensitive airway segmentation cnns by feature recalibration and attention distillation," in International Conference on Medical Image Computing and Computer-Assisted Intervention. Springer, 2020, pp. 221-231.

[35] H. Zheng, Y. Qin, Y. Gu, F. Xie, J. Yang, J. Sun, and G.-Z. Yanga, "Alleviating class-wise gradient imbalance for pulmonary airway segmentation," IEEE Transactions on Medical Imaging, 2021.

[36] W. Wu, Y. Yu, Q. Wang, D. Liu, and X. Yuan, "Upper airway segmentation based on the attention mechanism of weak feature regions," IEEE Access, vol. 9, pp. $95372-95381,2021$.

[37] Q. Xie, M.-T. Luong, E. Hovy, and Q. V. Le, "Self-training with noisy student improves imagenet classification," in Proceedings of the IEEE/CVF Conference on Computer Vision and Pattern Recognition, 2020, pp. $10687-10698$.

[38] H. Balacey, "Mise en place d'une chaîne complète d'analyse de l'arbre trachéo-bronchique à partir d'examen (s) issus d'un scanner-ct: de la 3d vers la 4d," Ph.D. dissertation, Bordeaux 1, 2013.

[39] P. Nardelli, K. A. Khan, A. Corvò, N. Moore, M. J. Murphy, M. Twomey, O. J. O'Connor, M. P. Kennedy, R. S. J. Estépar, M. M. Maher et al., "Optimizing parameters of an open-source airway segmentation algorithm using different ct images," Biomedical engineering online, vol. 14, no. 1, pp. 1-24, 2015.

[40] T. Inoue, Y. Kitamura, Y. Li, and W. Ito, "Robust airway extraction based on machine learning and minimum spanning tree," in Medical Imaging 2013: Computer-Aided Diagnosis, vol. 8670. International Society for Optics and Photonics, 2013, p. 86700L.

[41] A. Araujo, W. Norris, and J. Sim, "Computing receptive fields of convolutional neural networks," Distill, vol. 4, no. 11, p. e21, 2019.

[42] J. Hu, L. Shen, and G. Sun, "Squeeze-and-excitation networks," in Proceedings of the IEEE conference on computer vision and pattern recognition, 2018, pp. 7132-7141.

[43] C. Wei, K. Shen, Y. Chen, and T. Ma, "Theoretical analysis of self-training with deep networks on unlabeled data," arXiv preprint arXiv:2010.03622, 2020.

[44] F. Pérez-García, R. Sparks, and S. Ourselin, "Torchio: a python library for efficient loading, preprocessing, augmentation and patch-based sampling of medical images in deep learning," Computer Methods and Programs in Biomedicine, p. 106236, 2021.

[45] S. G. Armato III, G. McLennan, L. Bidaut, M. F. McNitt-Gray, C. R. Meyer, A. P. Reeves, B. Zhao, D. R. Aberle, C. I. Henschke, E. A. Hoffman et al., "The lung image database consortium (lidc) and image database resource initiative (idri): a completed reference database of lung nodules on ct scans," Medical physics, vol. 38, no. 2, pp. 915-931, 2011.

[46] A. Paszke, S. Gross, F. Massa, A. Lerer, J. Bradbury, G. Chanan, T. Killeen, Z. Lin, N. Gimelshein, L. Antiga et al., "Pytorch: An imperative style, high-performance deep learning library," Advances in neural information processing systems, vol. 32, 2019.

[47] Z. Xu, U. Bagci, B. Foster, A. Mansoor, J. K. Udupa, and D. J. Mollura, "A hybrid method for airway segmentation and automated measurement of bronchial wall thickness on ct," Medical image analysis, vol. 24, no. 1, pp. 1-17, 2015.

[48] F. Milletari, N. Navab, and S.-A. Ahmadi, "V-net: Fully convolutional neural networks for volumetric medical image segmentation," in 2016 fourth international conference on $3 D$ vision (3DV). IEEE, 2016, pp. 565-571.

[49] H. Chen, Q. Dou, L. Yu, J. Qin, and P.-A. Heng, "Voxresnet: Deep voxelwise residual networks for brain segmentation from $3 \mathrm{~d}$ mr images," NeuroImage, vol. 170, pp. 446-455, 2018.

[50] E. Smistad, A. C. Elster, and F. Lindseth, "Gpu accelerated segmentation and centerline extraction of tubular structures from medical images," International journal of computer assisted radiology and surgery, vol. 9, no. 4, pp. 561-575, 2014. 